\title{
A Low-Photocurrent CMOS Retinal Focal-Plane Sensor With a Pseudo-BJT Smoothing Network and an Adaptive Current Schmitt Trigger for Scanner Applications
}

\author{
Chung-Yu Wu, Fellow, IEEE, and Cheng-Ta Chiang, Student Member, IEEE
}

\begin{abstract}
In this work, a new structure of low-photocurrent CMOS retinal focal-plane sensor with pseudo-BJT smoothing network and adaptive current Schmitt trigger is proposed. The proposed structure is very simple and compact. This new circuit can easily be implemented in CMOS technology with a small chip area. Another innovation of this circuit is that the proposed circuit could be operated for low-induced current levels (pA), and the current hysteresis of the proposed current Schmitt trigger could be adjusted adaptively according to the value of induced photocurrents. In this work, the detection of static and moving objects, such as a moving white bar, are proven by projecting a pattern through HSPICE simulation. The proposed retinal focal-plane sensor includes a $32 \times 32$ pixel array with a pixel size of $70 \times 70 \mu \mathrm{m}^{2}$. The fill factor is $75 \%$ and the total chip area is $3000 \times 3030 \mu \mathrm{m}^{2}$. It is with fully functional $32 \times 32$ implementations consuming less than $8.8 \mu \mathrm{W}$ per pixel at 3.3 V. Measurement results show that the proposed new retinal focal-plane sensor has successfully been used in character recognition of scanner systems, such as pen scanners, etc.
\end{abstract}

Index Terms-BJT-based retinal sensor, CMOS smart sensor, neuromorphic vision chips, retinal processing circuit, VLSI.

\section{INTRODUCTION}

$\mathbf{T}$ RADITIONALLY, real-time vision proceeds by acquiring images with CCD cameras or CMOS imagers by processing them in digital platforms. The traditional approach to deal with vision tasks is to proceed according to two main steps: 1) acquisition of the image on a low-cost CCD camera and 2) software processing on digital platforms (DSP or PC). While the computational capabilities of digital platforms improve each year, they do not necessarily comply with low cost and portability. An attractive solution to this problem is to shift part of the computation into the sensor by dedicating some circuitry around photo-captor accomplished pixel-level processing. In this way, the CCD cameras or CMOS imagers are replaced with a more sophisticated device which is able to preprocess

Manuscript received August 24, 2003; revised January 11, 2004. This work was supported by the National Science Council, R. O. C., under contracts NSC91-2215-E-009-071. The associate editor coordinating the review of this paper and approving it for publication was Dr. Chang Liu.

The authors are with the Nanoelectronics and Gigascale Systems Laboratory, Department of Electronics Engineering and Institute of Electronics, National Chiao-Tung University, Hsinchu, 300, Taiwan, R.O.C. (e-mail: cywu@alab.ee.nctu.edu.tw; p9011838@alab.ee.nctu.edu.tw).

Digital Object Identifier 10.1109/JSEN.2004.828856 the acquired images and provide the next processing stages with limited requirements (computational power, low cost, portability, and low power consumption) [7]. Consequently, bio-inspired smart vision systems and applications become more and more attractive in research and development. The general approach in the design of these systems is to realize some processing as functions of neural vision systems of mammalians in the photo-sensor array [1]-[5] so that they can perform major functions in image processing, such as photo-input sensing, edge detection, moving object detection, etc.

As proposed in [3]-[5], the BJT-based retinal sensor chip mimics parts of functions of the cells in the outer plexiform layer of the real retina. As in the real retina, the retinal sensor chip similar advantageous features, such as high noise immunity, edge enhancement, and high dynamic range. Therefore, the BJT-based retinal structure [3]-[5] has been proven that it is very compact and suitable for VLSI implementation.

However, the parasitic p+-n-well-p-substrate BJTs used in the BJT-based retinal sensor have a smaller current gain as the n-well CMOS technology scaling down to $0.25 \mu \mathrm{m}$ or below [6]. Besides, the chip area of the parasitic BJT is large. To solve the problems mentioned above, a new circuit structure is developed and called the pseudo-BJT (PBJT) [6]. By incorporating a photodiode with the PBJT to form a photo-PBJT, the new PBJT-based retinal sensor with an adaptive current Schmitt trigger for noise removal is proposed. It has three advantages. First, the process-independent current gain could be precisely controlled by the geometric parameters of MOSFETs. Second, the chip area is less than that of the BJT-based retinal sensor. Third, the proposed circuit structure is compatible completely with advanced CMOS technology. Besides, the additional advantages of low operational photocurrent levels (pA) and robust noise immunity are proposed. In this work, this new PBJT-based retinal circuit is different from previous papers [3]-[6], [7]-[11]. Particularly, optical noise problems are more notable in recognition applied to characters, as mentioned in [13]. A new structure, the adaptive current Schmitt trigger, could be adjusted adaptively according to the value of induced photocurrents, which enhances noise immunity and eliminates disturbances. Since all MOS devices in the proposed retinal sensor circuit are operated in the subthreshold region, the power dissipation is very low. Thus, it is suitable for the front-end 
TABLE I

FEATURE COMPARISONS BETWEEN PBJT-BASED SILICON RETINA AND TYPICAL CMOS SENSOR

\begin{tabular}{|c|c|c|}
\hline $\begin{array}{c}\text { Feature } \\
\text { Comparisons }\end{array}$ & $\begin{array}{c}\text { PBJT-based } \\
\text { Silicon Retina }\end{array}$ & CMOS Sensor \\
\hline Technology & $\begin{array}{c}\text { TSMC } 0.35 \mu \mathrm{m} \\
\text { IP4M }\end{array}$ & $\begin{array}{c}\text { TSMC } 0.35 \mu \mathrm{m} \\
\text { IP4M }\end{array}$ \\
\hline Pixel size & $\begin{array}{c}70 \mu \mathrm{m} \times 70 \mu \mathrm{m} \text { (fill } \\
\text { factor } 75 \% \text { ) }\end{array}$ & $\begin{array}{c}7 \text { um } x>\mu \mathrm{m} \text { (fill } \\
\text { factor } 30 \%)\end{array}$ \\
\hline $\begin{array}{l}\text { Photosensitivity per } \\
\text { photodio de area }\end{array}$ & $120 \mu \mathrm{A} / \mathrm{cm}^{2}-\operatorname{lux}$ & $6 \mu \mathrm{A} / \mathrm{cm}^{2}-\mathrm{lux}$ \\
\hline $\begin{array}{c}\text { Stan dby dark } \\
\text { power consumption }\end{array}$ & $\sim 0$ & $10 \mathrm{~mW}$ \\
\hline $\begin{array}{c}\text { Operational pixel } \\
\text { power consumption }\end{array}$ & $\begin{array}{c}8.8 \mu W / p i x e l \\
\text { (Array size } 32 \times 32)\end{array}$ & $\begin{array}{c}0.32 \mu W / p i x e l \\
\text { (Array size } 352 \times 288)\end{array}$ \\
\hline Post proc essor & $\begin{array}{l}\text { Simple (without } \\
\text { noise removal } \\
\text { operation) }\end{array}$ & Complex \\
\hline $\begin{array}{c}\text { Optical dynamic } \\
\text { range }\end{array}$ & $120 \mathrm{~dB}$ & $70 \mathrm{~dB}$ \\
\hline Noise immunity & Yes & None \\
\hline Processing time & Real-time & $\begin{array}{l}\text { Need integration } \\
\text { time about } 30 \mathrm{~ms}\end{array}$ \\
\hline Timing diagram & The same & The same \\
\hline Power supply & $3.3 \mathrm{~V}$ & $3.3 \mathrm{~V}$ \\
\hline Scalable & Yes & Yes \\
\hline Image output & Digital & Analog \\
\hline $\begin{array}{c}\text { Sensor } \\
\text { characteristics }\end{array}$ & Intelligent & Regular \\
\hline
\end{tabular}

sensor applications of portable optical scanner systems like pen scanners [14]. Table I shows the feature comparisons between PBJT-based retinal sensor and typical CMOS sensor. Proven by Table I, these superior features of retinal processing circuits to the conventional CMOS and CCD images are the same as mentioned in [15]. This new PBJT-based retinal sensor has excellent photosensitivity per photodiode area, optical dynamic range, noise immunity, continuous sensing, edge enhancement, and intelligent characteristic.

In this work, the detection of static and moving objects, such as a moving white bar, are tested and proved through HSPICE simulation. The area of this chip is $3000 \times 3030 \mu \mathrm{m}^{2}$ and that of a single pixel is $70 \times 70 \mu \mathrm{m}^{2}$ with a fill factor of $75 \%$. It is with fully functional $32 \times 32$ implementations consuming less than $8.8 \mu \mathrm{W}$ per pixel at $3.3 \mathrm{~V}$. Measurement results successfully verified the correct functions and performance of the proposed new retinal sensor circuits in character recognition of scanner systems. This proposed retinal chip could recognize the size of characters capable of scanning, which is $8-14$ points. The screen size is $32 \times 32$ pixels, and the scanner resolution is $169 \mathrm{dpi}$ (14 pixels $/ \mathrm{mm}$ ). The illumination for all the following measurements is set at 0.87 normalized with fluorescent light at a wavelength of $550 \mu \mathrm{m}$ (13.8 lux).

In Section II, it describes the architecture and the proposed circuit. Section III presents simulation results of the proposed circuit. Section IV displays measurement results. Section V gives conclusions and feature works.

\section{ARChitecture AND CirCUIT}

Fig. 1 shows the architecture of the proposed retinal focalplane sensor, which consists of the $32 \times 32$ pixel array and the

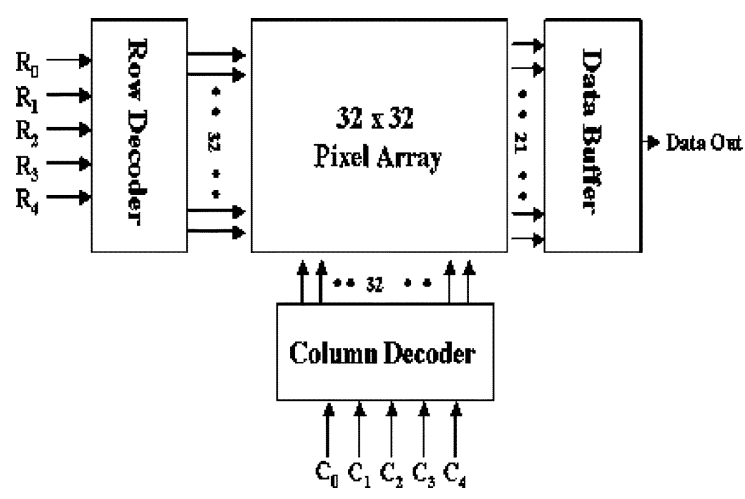

Fig. 1. Architecture of the proposed retinal focal-plane sensor.

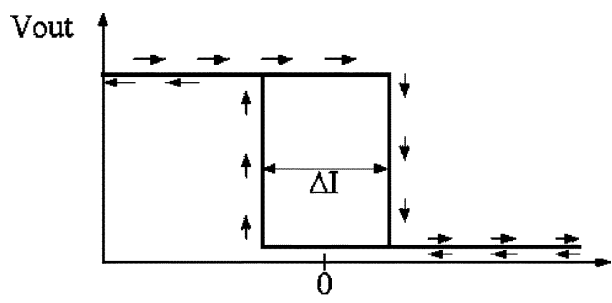

Iisolation -Ismooth

Fig. 2. Concept of adaptive current Schmitt trigger of proposed retinal focal-plane sensor.

peripheral circuits. First, the pixel array is to detect the image with noise and function for noise removal. Next, the row and column decoder could select the desired pixel randomly. Finally, the data buffer is to convert the parallel data to serial data in order to reduce the output pins of the chip.

Fig. 2 shows the conceptual dc output characteristics of the adaptive current Schmitt trigger. Current hysteresis $(\Delta \mathrm{I})$ is the quality of the current Schmitt trigger in which the input threshold changes as a function of the input current level. Adding current hysteresis in the retinal sensor circuit could enhance the function of noise immunity. As the input starts positive and goes negative, the output does not change until it reaches the negative trip point. In the same way, the input starts negative and goes positive in direction, the output does not switch until it reaches the positive trip point. In the proposed pixel structure of a retinal focal-plane sensor, as shown in Fig. 3, an isolated PNP pseudo-BJT is used as photoreceptor, a smoothing NPN pseudo-BJT with adjustable N-channel MOSFET resistors is used to form the retinal smoothing network, an adaptive current Schmitt trigger, and an inverter are included. The transistors $\mathrm{Mp} 1, \mathrm{Mp} 2$, and photodiode $\mathrm{D}_{0}$ are as the PNP pseudo-BJT. The transistors Mn1, Mn2, and a photodiode $\mathrm{D}_{0}$ are as the NPN pseudo-BJT with four adjustable $\mathrm{N}$-channel MOS resistors Ms1-Ms4 as the smoothing network. The smoothing network is connected to its four neighbors, and the resistance of four MOS resistors is controlled by the gate voltage Vsmooth (VF). The adaptive current Schmitt trigger comprises of Mp1, Mp2, Mn1, and Mn2, and hysteresis level adjustment Mpf1, Mpf2, Mnf1, and Mnf2. The current Iiso is the generated current of PNP pseudo-BJT whereas the current Ismt is that of NPN pseudo-BJT. In this proposed retinal sensor, it operates in the subthreshold region. Therefore, the circuit just consumes little power during in nonlighting mode. If the 


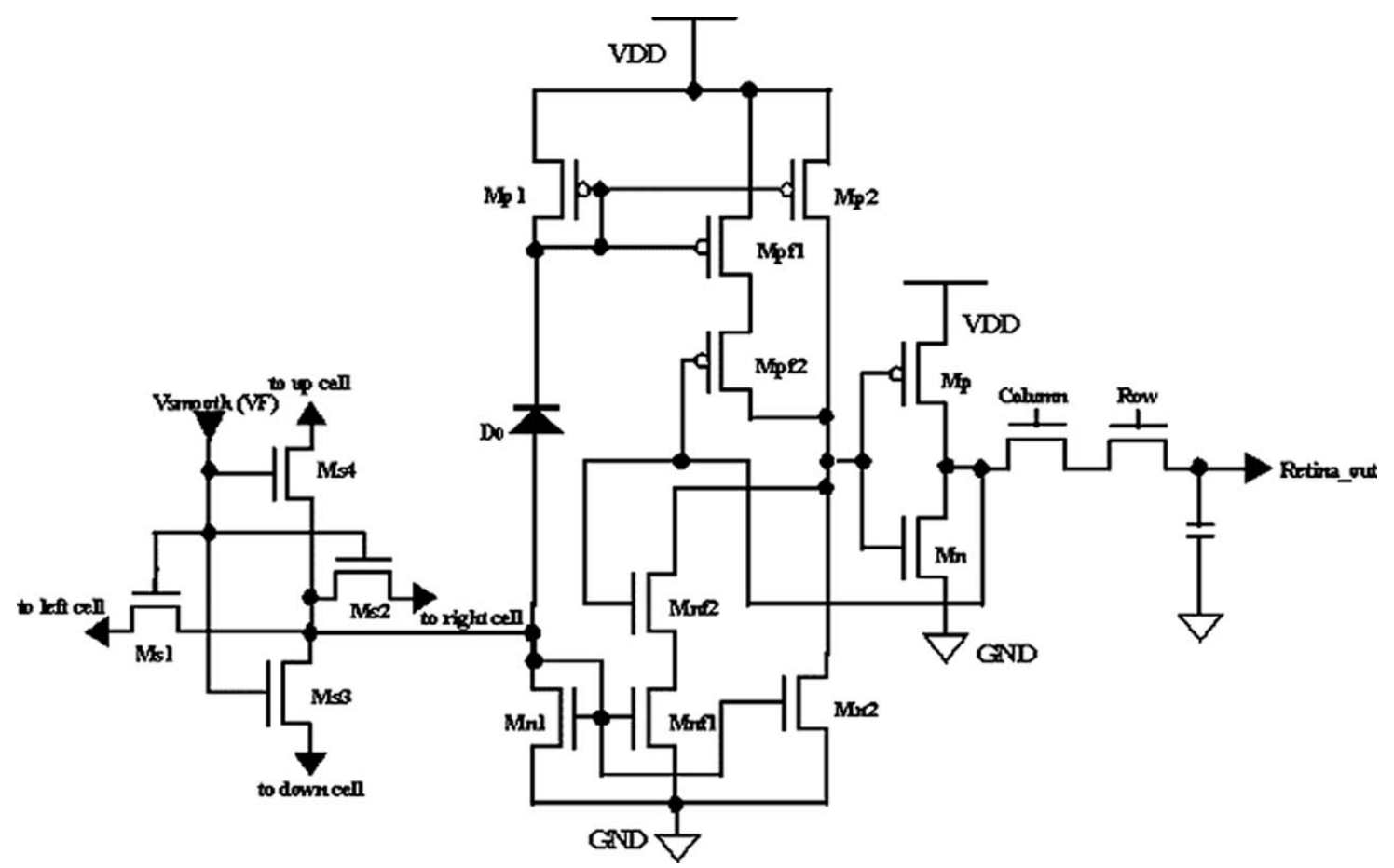

Fig. 3. Pixel structure of the proposed retinal focal-plane sensor circuit.

TABLE II

Designed DeVICE Dimensions AND the CONTROLLING Voltages OF THE PiXel CiRCUIT IN Fig. 3

\begin{tabular}{|c|c|}
\hline Technology & $0.35 \mu \mathrm{m}$ SLICDE CMOS (SPQM) \\
\hline Mpl (WL) & $\mathrm{l} \mu \mathrm{m} / \mathrm{l} \mu \mathrm{m}$ \\
\hline$\overline{M p 2 ~(W L) ~}$ & $20 \mu \mathrm{m} / 1 \mu \mathrm{m}$ \\
\hline$\overline{M p f 1}(W / L)$ & $2 \mu \mathrm{m} / 1 \mu \mathrm{m}$ \\
\hline Mpf2 (WiL) & $2 \mu \mathrm{m} / 1 \mu \mathrm{m}$ \\
\hline$\overline{\operatorname{Mnl}}(\mathrm{WL})$ & $\mathrm{l} \mu \mathrm{m} / \mathrm{l} \mu \mathrm{m}$ \\
\hline$\overline{M n 2 ~(W L) ~}$ & $20 \mu \mathrm{m} / 1 \mu \mathrm{m}$ \\
\hline$\overline{M n f 1}(W / L)$ & $10 \mu \mathrm{m} / 1 \mu \mathrm{m}$ \\
\hline$\overline{M n 2}(W / L)$ & $10 \mu \mathrm{m} / 1 \mu \mathrm{m}$ \\
\hline $\bar{M} s \mathrm{l}=\mathrm{Ms} 2=\mathrm{M} s \mathrm{~s}=\mathrm{M} s 4$ (W/L) & $\mathrm{l} \mu \mathrm{m} / \mathrm{l} \mu \mathrm{m}$ \\
\hline$\overline{M p}(\mathrm{~W} / \mathrm{L})$ & $0.4 \mu \mathrm{m} / 0.35 \mu \mathrm{m}$ \\
\hline$\overline{M n}(W /)$ & $0.4 \mu \mathrm{m} / 0.35 \mu \mathrm{m}$ \\
\hline$V_{\text {smooth }}(V F)$ & Adjustable \\
\hline
\end{tabular}

current Ismt is bigger than the current Iiso, at first, the Vout (Retina_out) goes too high and it turns on the MOS Mnf2 to draw the current $\Delta \mathrm{I} / 2$. By the same token, the current Ismt is smaller than the current Iiso initially, the Vout (Retina_out) stays low level and it turns on the MOS Mpf2 to sink the current $\Delta \mathrm{I} / 2$. The positive and negative trip points can be expressed as

$$
\text { Iiso }- \text { Ismt }= \pm \frac{\Delta \mathrm{I}}{2}
$$

where $\Delta \mathrm{I}$ is the current hysteresis. If the induced photocurrent is larger, the current of transistors $\mathrm{Mn} 1$ and Mp1 becomes also larger. Due to the function of current mirror Mn1-Mnf1 and Mp1-Mpf1, the current of Mnf1 and Mpf1 could be adjusted by the induced photocurrent. Hence, this proposed circuit could adjust the current $\Delta \mathrm{I}$ adaptively without external controlling voltage. The transistors $\mathrm{Mp}$ and $\mathrm{Mn}$ are composed of an inverter to amplify the output of the adaptive current Schmitt trigger to VDD or GND so that the signal is converted from analog to bi-

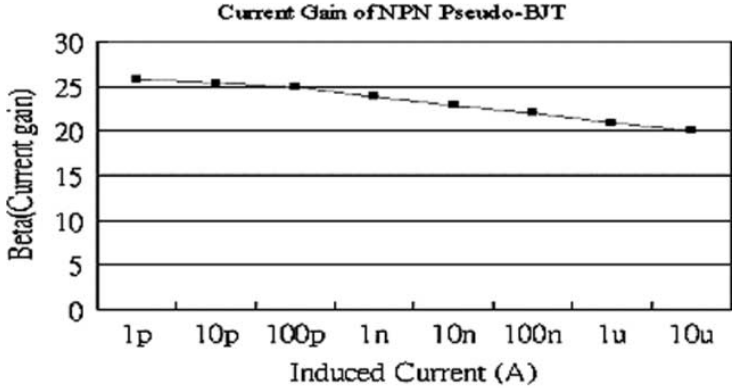

(a)

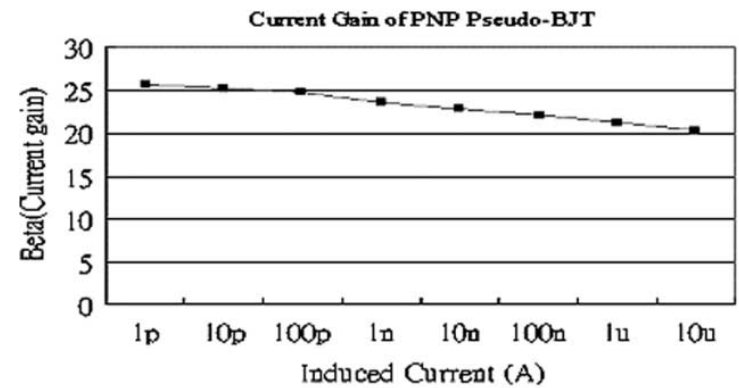

(b)

Fig. 4. (a) Current gain of NPN pseudo-BJT. (b) Current gain of PNP pseudo-BJT.

nary. All the simulations of the proposed circuit are analyzed in Section III.

\section{Simulation RESUlTS}

All simulation results are based upon the device parameters of $0.35-\mu \mathrm{m}$ 1P4M CMOS technology with $3.3-\mathrm{V}$ power supply. The designed device dimensions and the controlling voltage of the pixel circuit in Fig. 3 are listed in Table II. Fist, we simulate the current gain of NPN pseudo-BJT and PNP pseudo-BJT in 

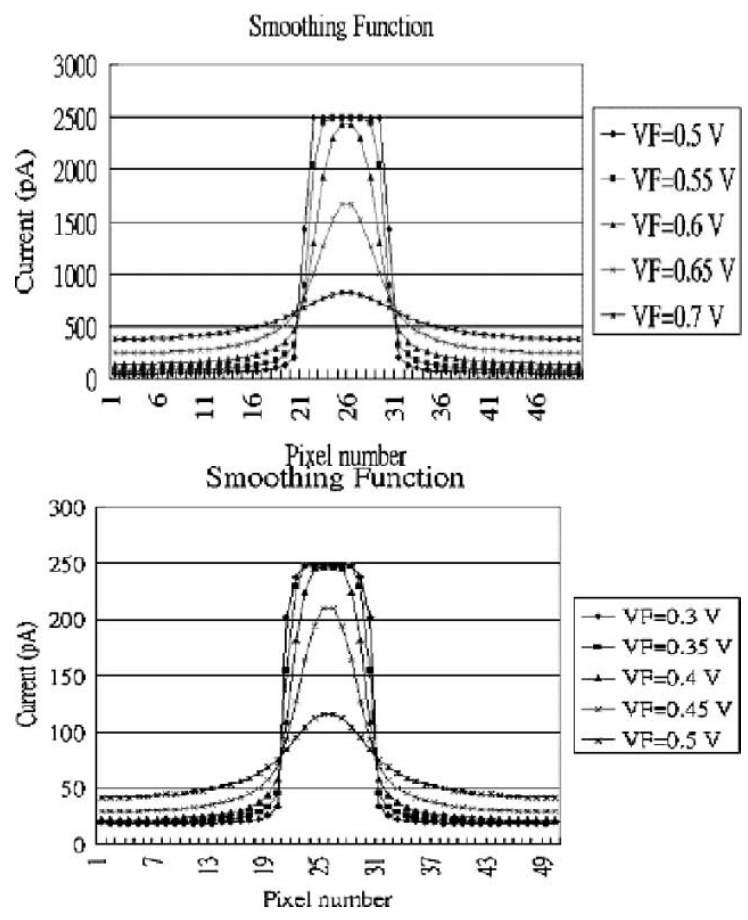

Fig. 5. (a) Smoothing function for induced photocurrent $100 \mathrm{pA}$. (b) Smoothing function for induced photocurrent $10 \mathrm{pA}$.

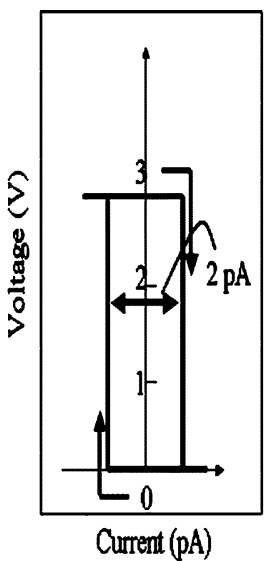

(a)

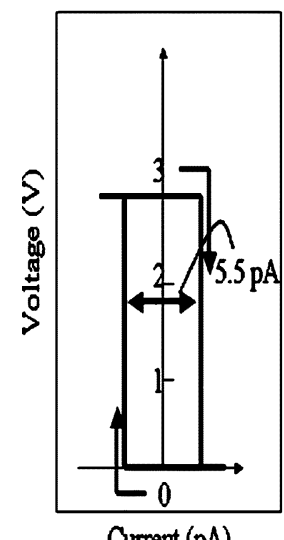

(c)

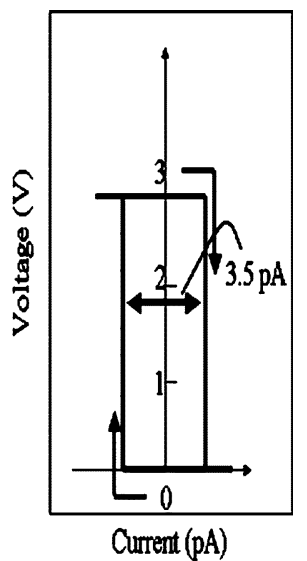

(b)

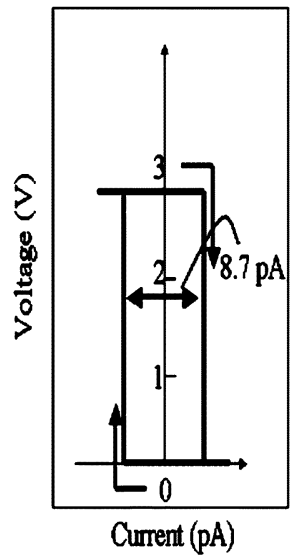

(d)

Fig. 6. DC output characteristics (a)-(d) of adaptive current Schmitt trigger with fixed Ismt current 10 pA, 20 pA, 30 pA, and 40 pA, respectively.
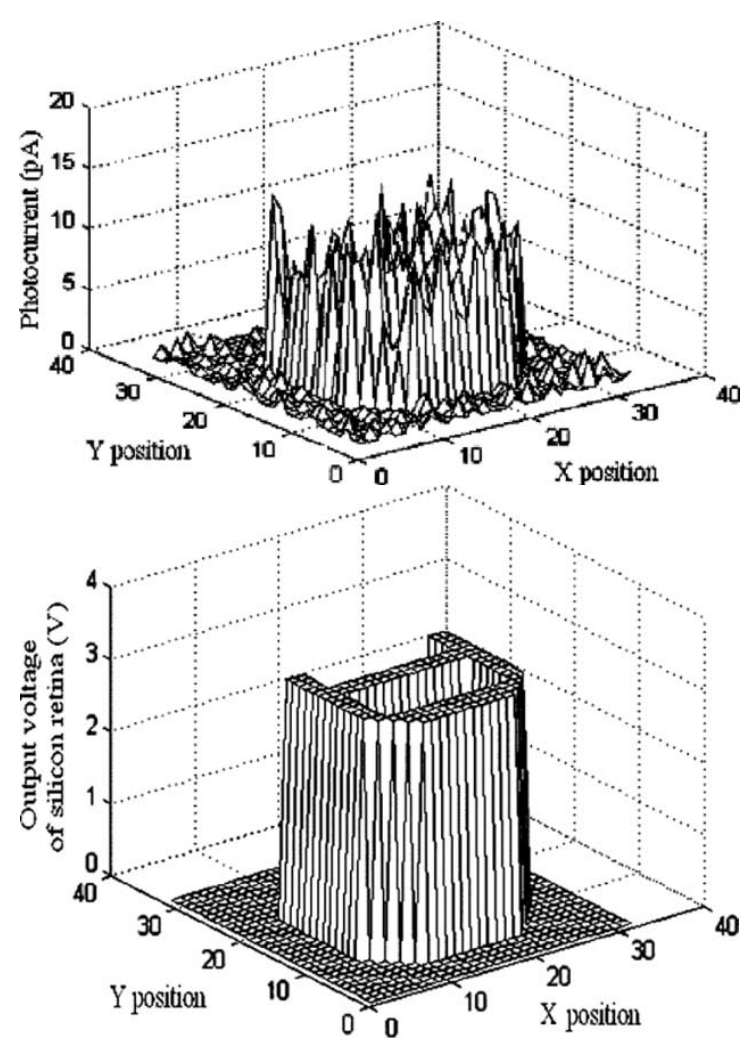

Fig. 7. (a) Input pattern A with a zero mean random noise that is with a standard deviation of $60 \%$ of induced current and the induced photocurrent 10 pA. (b) $32 \times 32$ pixels output (Retina_out) of 2-D retinal focal-plane sensor for input pattern A.

the low-induced current level, respectively. The drain current equation in the subthreshold region can be expressed as [12]

$$
\mathrm{Ids}=\mathrm{K}_{\mathrm{x}} \frac{\mathrm{W}}{\mathrm{L}}\left[1-\exp \left(\frac{-\mathrm{V}_{\mathrm{DS}}}{\mathrm{vt}}\right)\right] \exp \left[\frac{\left(\mathrm{V}_{\mathrm{GS}}-\mathrm{Vth}\right)}{\mathrm{nvt}}\right]
$$

where $\mathrm{K}_{\mathrm{x}}$ depends on process parameter, $\mathrm{W} / \mathrm{L}$ is the geometric ratio of the MOS, Vth is the threshold voltage of MOS, vt is the thermal voltage and is given by Kt/q, and $\mathrm{n}$ is the subthreshold swing parameter. Based on (2), the current gain of pseudo-BJT can be written as

$$
\begin{aligned}
\beta=\frac{\left(\frac{\mathrm{W}}{\mathrm{L}}\right) 1}{\left(\frac{\mathrm{W}}{\mathrm{L}}\right) 2} \frac{[1}{[1} & -\exp \left(\frac{\left.\left.-\mathrm{V}_{\mathrm{DS} 1}\right)\right]}{\mathrm{vt}}\right) \\
& \exp \left(\frac{\left.\left.-\mathrm{V}_{\mathrm{DS} 2}\right)\right]}{\mathrm{vt}}\right) \\
& \times \exp \left[\left(\frac{1}{n 1}-\frac{1}{n 2}\right)\left(\frac{\left(\mathrm{V}_{\mathrm{GS}}-\mathrm{Vth}\right)}{\mathrm{vt}}\right)\right] .
\end{aligned}
$$

By using (3), in Fig. 4, it shows the beta value of NPN pseudo-BJT and PNP pseudo-BJT. Due to the parameter of $\mathrm{V}_{\mathrm{DS}}$ for master and slave MOSFETs, in low-induced current levels, the current gain is bigger than the designed value. When the induced current becomes bigger, the parameter $V_{D S}$ for master and slave MOSFETs are close to each other. Therefore, the current gain approaches to desired value as shown in Fig. 4. In order to reduce the affect of subthreshold swing parameters, the slave MOSFETs must be structured by making parallel copies of the master MOSFET. Hence, the current gain would be independent of the subthreshold swing parameter. Next, it is applied in a one-dimensional (1-D) array to verify the function 


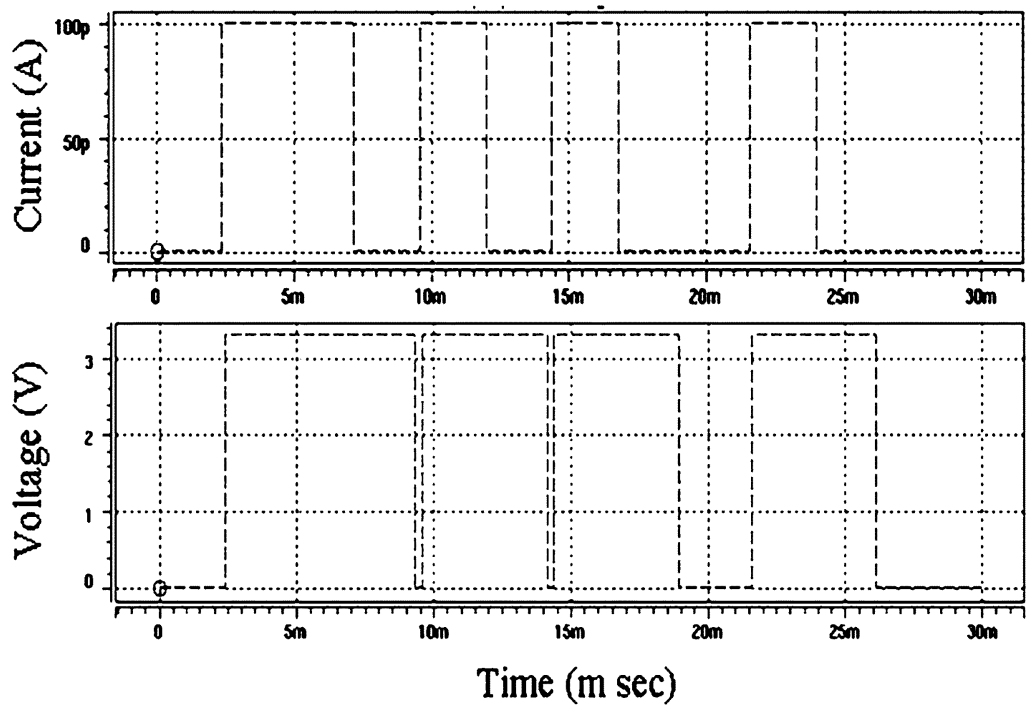

Fig. 8. Output voltages under input current waveforms corresponding to the photocurrents generated by moving image patterns of white bars with minimum width of $500 \mu \mathrm{m}$.

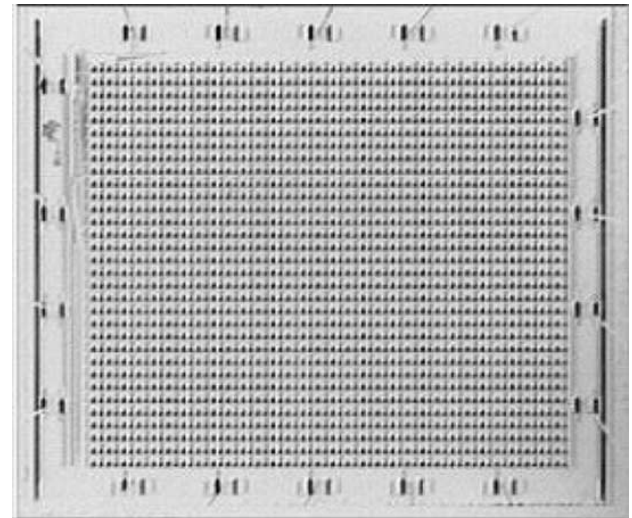

(a)

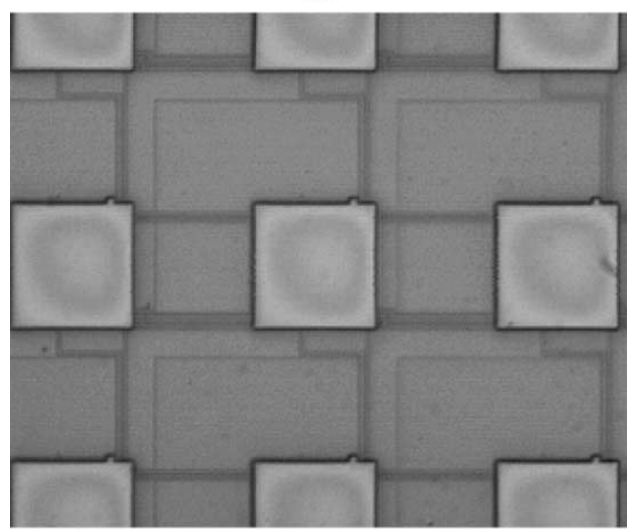

(b)

Fig. 9. Photographs of the proposed retinal sensor for (a) the whole fabricated chip and (b) a single pixel.

of smoothing, as shown in Fig. 5(a) and (b), with 50 pixels is considered. The light is incident on the 20th to the 30th pixels. Finally, to verify the performance of circuits for low induced current levels, the induced photocurrent is simulated in 100 pA, and 10 pA, respectively. Compared to Fig. 5(a) and (b), the main difference between them is the VF. If the incident lighting were larger, the photodiode of the retinal sensor would

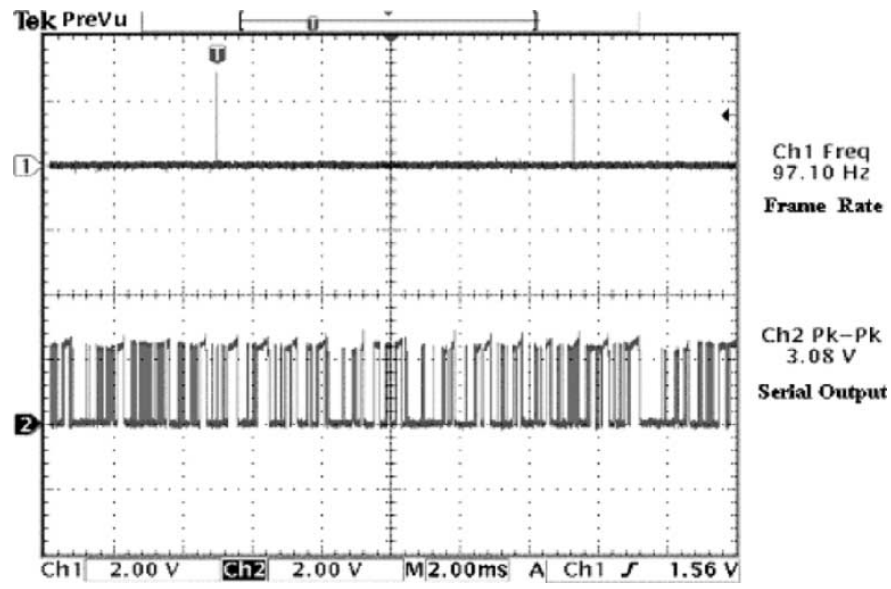

Fig. 10. Measured waveforms for frame rate ( 97.1 frames/s) and serial output via data buffer.

induce bigger currents. Therefore, the resistance of smoothing networks could be smaller because of the suitable scale of voltage variations in the smoothing network. In other words, if it is in the dimming-illumination environment, the induced photocurrent is smaller. Therefore, it needs bigger resistance to function as expected. The MOS resistance in the subthreshold region can be expressed as

$$
\operatorname{Rds}=\left[\mathrm{Kx} \frac{\mathrm{W}}{\mathrm{L}} \exp \left[\frac{(\mathrm{VF}-\mathrm{Vth})}{n v_{\mathrm{t}}}\right]\right]^{-1} .
$$

As seen from (4), Rds is tunable by VF. Thus, the smoothing characteristics of retinal sensors can be adjusted by VF. In these simulation results, as mentioned above, this proposed circuit could be operated in the dimming-illumination environment for low induced current levels (pA). In Fig. 6, the dc output characteristics (a)-(d) of the adaptive current Schmitt trigger with fixed Ismt current is shown. This dc characteristic is to verify the function of adaptive current Schmitt trigger, which is to fix the Ismt current and to sweep the Iiso current. For the fixed smoothing current (a)-(d) in Fig. 6, they are 10 pA, 20 pA, 30 pA, and 40 


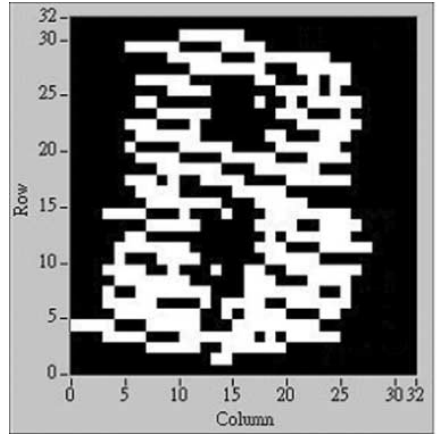

(a)

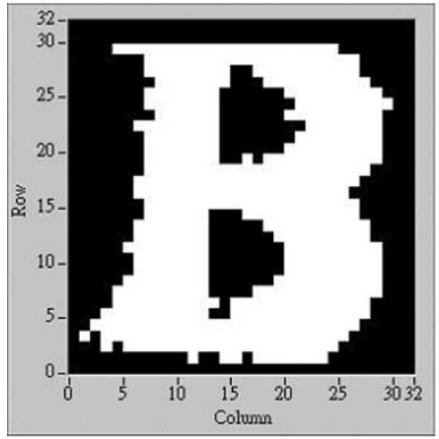

(d)

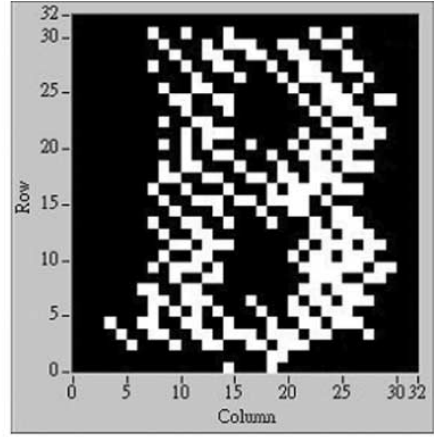

(b)

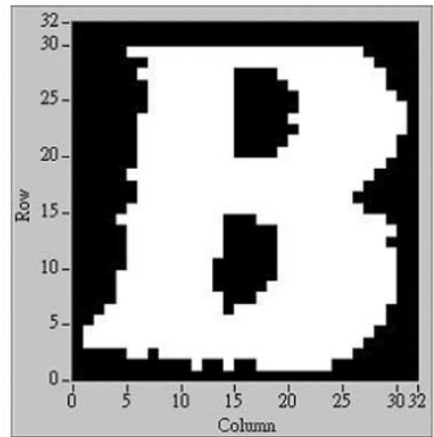

(e)

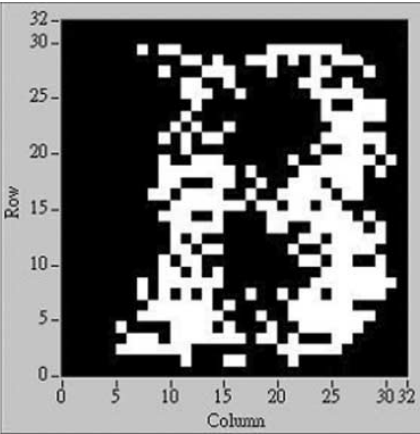

(c)

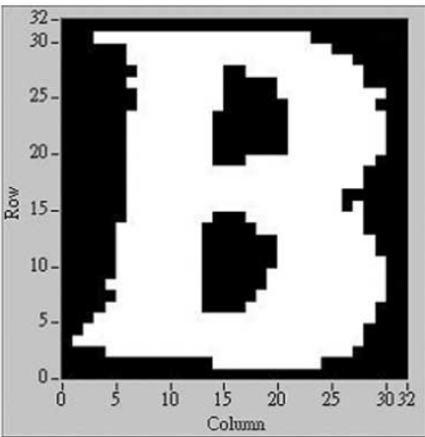

(f)

Fig. 11. Measurement results normalized with fluorescent light at wavelength of $550 \mu \mathrm{m}$ (13.8 lux) of different illuminations for character "B" at (a) 0.02, at (b) 0.04 , at (c) 0.54 , at (d) 0.87 , at (e) 2.65 , and at (f) 5.3 .

pA, respectively. Based on (1), the center point is the position of the smoothing current Ismt and the dc current output is symmetric, as shown in Fig. 2. The values of $\Delta \mathrm{I}$ are $2 \mathrm{pA}, 3.5 \mathrm{pA}$, $5.5 \mathrm{pA}$, and $8.7 \mathrm{pA}$, respectively. Therefore, the simulation results show that current hysteresis could be adjusted adaptively according to the value of induced photocurrent.

Finally, we apply this retinal focal-plane sensor into two-dimensional (2-D) $32 \times 32$ pixel array, and simulate the incident static image to verify the detecting function of 2-D retinal focal-plane sensor. In Fig. 7(a), the input pattern A with noise, which is a zero-mean random noise with a standard deviation of $60 \%$ of induced photocurrent, is shown. Fig. 7(b) presents the $32 \times 32$ pixels output (Retina_out) of the 2-D retinal focal-plane sensor. Thus, good performance for detection on the 2-D retinal sensor is illustrated. Moreover, due to the pseudo-BJT smoothing network and adaptive current Schmitt trigger, these simulation results show robust noise immunity in this proposed circuit. In Fig. 8, the SPICE simulated output voltages under input current waveforms corresponding to the photocurrents generated by moving image patterns of white bars with a minimum width of $500 \mu \mathrm{m}$ where the scanning speed is $100 \mathrm{~mm} / \mathrm{s}$, which is larger than the maximum specified speed of $40 \mathrm{~mm} / \mathrm{s}$ for pen scanners. These simulation results have successfully confirmed the correct functions and performance of the proposed new retinal sensor circuits in the character recognition of scanner systems.

\section{MEASUREMENT Results}

Fig. 9(a) presents a photograph of the layout of the fabricated chip and Fig. 9(b) displays the photograph of a single pixel.

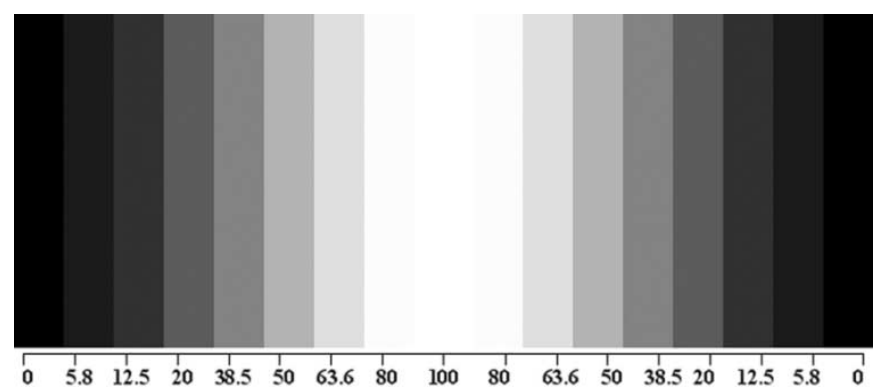

(a)

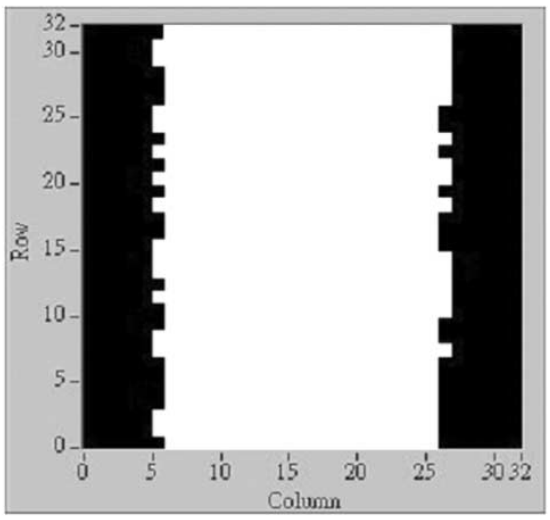

(b)

Fig. 12. (a) Static-graded image pattern with different contrasts (\%) incident upon the retinal sensor. (b) The measurement results for enhanced edges with the minimum acceptable contrast of $20 \%$.

The area of this chip is $3000 \times 3030 \mu \mathrm{m}^{2}$ and that of a single pixel is $70 \times 70 \mu \mathrm{m}^{2}$ with a fill factor of $75 \%$ and with fully 


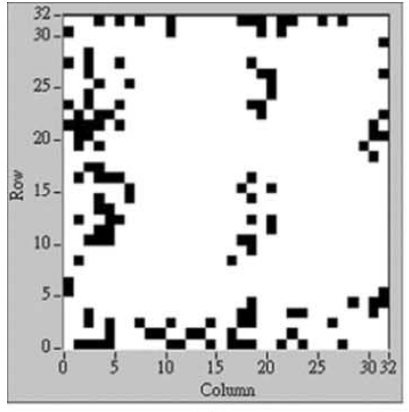

(a)

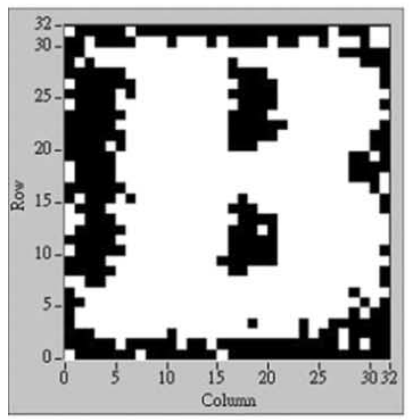

(c)

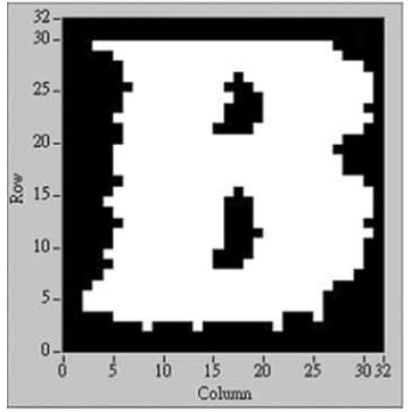

(e)

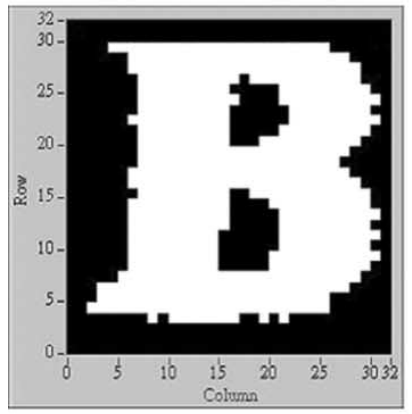

(g)

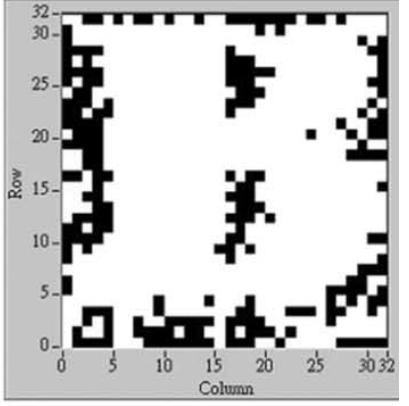

(b)

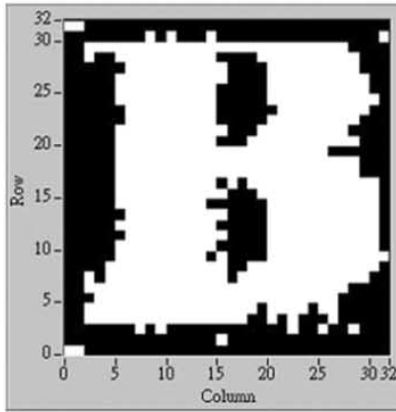

(d)

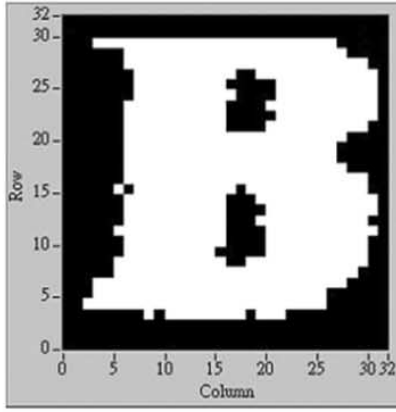

(f)

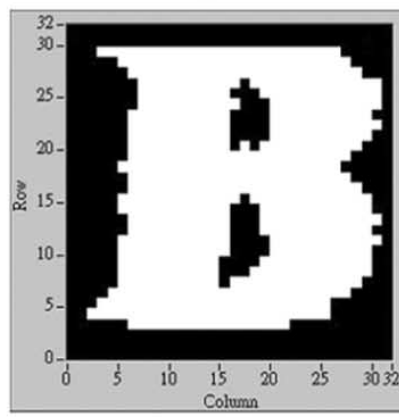

(h)
Fig. 13. Measurement results of the smoothing function for noise removal at different control voltage for character " $\mathrm{B}$ " at (a) $\mathrm{VF}=0.85 \mathrm{~V}$, at (b) $\mathrm{VF}=0.9 \mathrm{~V}$, at (c) $\mathrm{VF}=0.95 \mathrm{~V}$, at (d) $\mathrm{VF}=1 \mathrm{~V}$, at (e) $\mathrm{VF}=1.1 \mathrm{~V}$, at (f) $\mathrm{VF}=1.2 \mathrm{~V}$, at (g) $\mathrm{VF}=1.4 \mathrm{~V}$, at $(\mathrm{h}) \mathrm{VF}=1.5 \mathrm{~V}$.

functional $32 \times 32$ implementations consuming less than $8.8 \mu \mathrm{W}$ per pixel at $3.3 \mathrm{~V}$. Fig. 10 shows the oscilloscope traces of frame rates for fully serial readouts and serial output via the data buffer. The frame rate for character reorganization is 97.1 frames/s. Fig. 11(a)-(f) display measurement results normalized with fluorescent lights at a wavelength of 550 $\mu \mathrm{m}$ (13.8 lux) of different illuminations for character " $\mathrm{B}$," which is in the range of $0.02-5.3$. Fig. 12(a) is the static

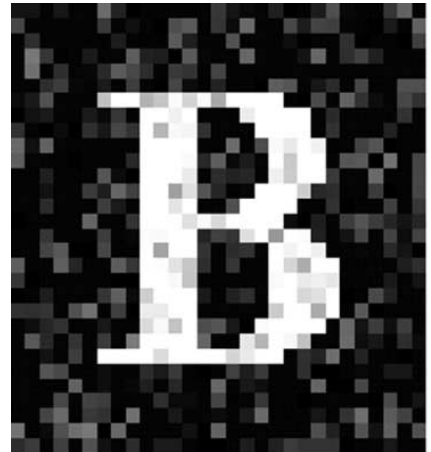

(a)

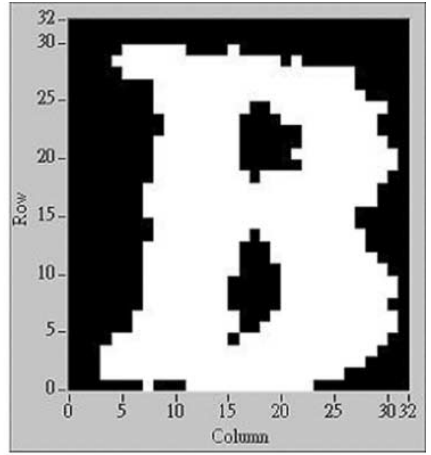

(b)
Fig. 14. (a) Incident image with random distributed noise with a standard deviation of $50 \%$. (b) The measurement result at $\mathrm{VF}=1.5 \mathrm{~V}$.

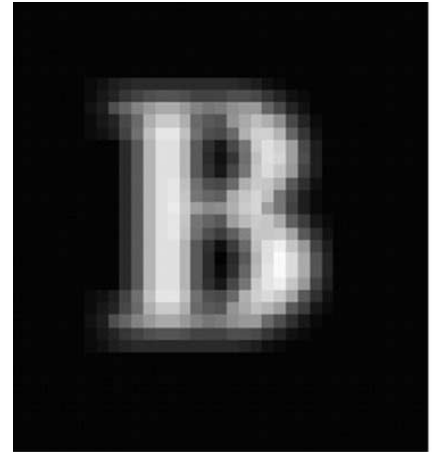

(a)

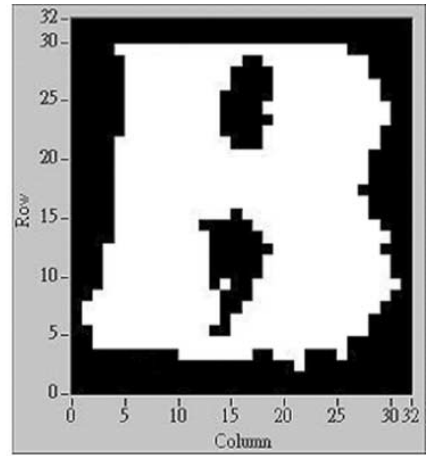

(b)
Fig. 15. (a) Incident image blurred. (b) The measurement result at VF $=1.5 \mathrm{~V}$.

graded image pattern with different contrasts (\%) fully incident upon the retinal sensor. The contrast for whole black and complete white is $100 \%$. In Fig. 12(b), the contrast of image is enhanced and columns 6 and 26 are located in the contrast of $20 \%$, as shown in Fig. 12(a). Therefore, the minimum acceptable contrast is 20\%. Fig. 13(a)-(h) successfully show the smoothing function for noise removal at different control voltages, which range from $\mathrm{VF}=0.85-1.5 \mathrm{~V}$. Fig. 14(a) presents the incident image with random distributed noise with a standard deviation of $50 \%$ and Fig. 14(b) is the measurement result at $\mathrm{VF}=1.5 \mathrm{~V}$. Fig. 15(a) shows the incident image blurred and Fig. 15(b) is the measurement result at $\mathrm{VF}=1.5 \mathrm{~V}$. The measurement results of Fig. 14(b) and Fig. 15(b) show that the proposed new retinal sensor circuits could restore unclear characters, as show in Fig. 14(a) and Fig. 15(a), into clear characters. Fig. 16 shows the minimum black space versus the scanning speed for character patterns in $169 \mathrm{dpi}$. The minimum adjacent distance is expected to be increased with scanner speed, and this is observed. The measurement results for this proposed retinal chip could recognize the size of characters capable of scanning, which is 8-14 points. The screen size is $32 \times 32$ pixels, and the scanner resolution is $169 \mathrm{dpi}$ (14 pixels/mm). The illumination for all of the following measurements is set at 0.87 normalized with fluorescent light at a wavelength of $550 \mu \mathrm{m}$ (13.8 lux). These measurement results have successfully verified the correct functions and performance of the proposed new 


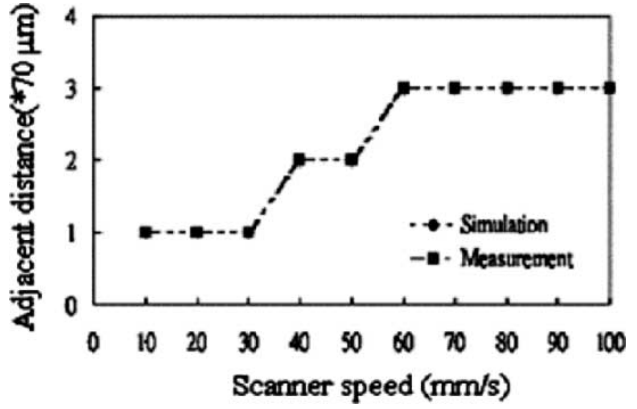

Fig. 16. Scanner speed and minimum adjacent distance.

TABLE III

SUMMARY ON THE CHARACTERISTICS OF THE FABRICATED PBJT-BASED RETINAL SENSOR FOR SCANNER APPLICATIONS

\begin{tabular}{l|l}
\hline Technology & $0.35 \mu \mathrm{m}$ SLICDE CMOS (SPQM) \\
\hline Resolution & $32 \times 32$ \\
\hline Sampling geometry & Rectangular \\
\hline Fixel size & $70 \mu \mathrm{m} \times 70 \mu \mathrm{m}$ \\
\hline Fill factor (size of light window) & $75 \%$ \\
\hline Chip size & $3000 \mu \mathrm{m} \times 3030 \mu \mathrm{m}$ \\
\hline Power supply & $3.3 \mathrm{~V}$ \\
\hline Total dc power dissipation & $9 \mathrm{~mW}$ \\
\hline Current gain of PBJTs & 20 \\
\hline Readout time of one pixel & $10.4 \mu \mathrm{s}$ \\
\hline Frame rate for total serial readout & $97.1 \mathrm{frames} / \mathrm{s}$ \\
\hline Scanner resolution & $169 \mathrm{dpi}$ \\
\hline Size of characters capable of scanning & $8-14$ \\
\hline
\end{tabular}

retinal sensor circuits in the character recognition of scanner systems. The characteristics of the fabricated PBJT-based retinal sensor for scanner applications are summarized in Table III.

\section{CONCLUSION}

This work presents a low-photocurrent CMOS retinal focalplane sensor with a pseudo-BJT smoothing network and adaptive current Schmitt trigger. In this proposed architecture, the pixel structure is very simple and compact compared to the BJT-based retinal sensor. Due to the pseudo-BJT smoothing network and adaptive current Schmitt trigger, these measurement results show robust noise immunity in this proposed circuit. The minimum acceptable contrast is $20 \%$. The screen size is $32 \times 32$ pixels and the scanner resolution is $169 \mathrm{dpi}$ ( 14 pixels $/ \mathrm{mm})$. The area of this chip is $3000 \times 3030 \mu \mathrm{m}^{2}$ and that of a single pixel is $70 \times 70 \mu \mathrm{m}^{2}$ with a fill factor of $75 \%$ and with fully functional $32 \times 32$ implementations consuming less than $8.8 \mu \mathrm{W}$ per pixel at $3.3 \mathrm{~V}$. According to the measurement results above, this work has successfully verified the correct functions and performance of the proposed new retinal sensor circuits in character recognition of scanner systems. In future research, this fabricated chip will be adaptively adjusted according to desired applications, for example, applied in surveillance sensor systems or intelligent transportation systems, etc.

\section{ACKNOWLEDGMENT}

The authors would like to thank the AlfaPlus Semiconductor Corporation for their help with chip fabrication.

\section{REFERENCES}

[1] E. Funatsu, Y. Nitta, Y. Miyake, T. Toyoda, J. Ohta, and K. Kyuma, "An artificial retina chip with current-mode focal plane image processing functions," IEEE Trans. Electron Devices, vol. 44, pp. 1777-1781, Oct. 1997.

[2] R. A. Panicacci, S. E. Kemeny, P. D. Jones, C. Staller, and E. R. Fossum, "128 Mb/s multiport CMOS binary active-pixel image sensor," in ISSCC Dig. Tech. Papers, 1996, pp. 100-101.

[3] C. Y. Wu and C. F. Chiu, "A new structure of 2-D silicon retina," IEEE J. Solid-State Circuits, vol. 30, pp. 890-897, Aug. 1995.

[4] C. Y. Wu and H. C. Jiang, "An improved BJT-based silicon retina with tunable image smoothing capability," IEEE Trans. VLSI, vol. 7, pp. 241-248, June 1999.

[5] H. C. Jiang and C. Y. Wu, "A 2-D velocity- and direction-selective sensor with BJT-based silicon retina and temporal zero-crossing detector," IEEE J. Solid-State Circuits, vol. 34, pp. 241-247, Feb. 1999.

[6] C. Y. Wu, H. C. Huang, L. J. Lin, and K. H. Huang, "A new PseudoBipolar-Junction-Transistor (PBJT) and its application in the design of retinal smoothing network," in Proc. IEEE Int. Symp. Circuits and Systems, vol. 4, July 2002, pp. 125-128.

[7] P. Y. Burgi and F. Heitger, "A fast $100 \times 100$ pixel silicon retina for edge extraction with application in OCR," in Proc. IEEE Int. Symp. Signal Processing and Its Applications, vol. 37, 2001, pp. 288-291.

[8] G. Liang and W. C. Miller, "A novel photo BJT array for intelligent imaging," in Proc. IEEE Int. Symp. Circuits and System Systems, vol. 2, Aug. 1993, pp. 1056-1059.

[9] M. D. Rowley and J. G. Harris, "An edge enhancement technique for analog VLSI early vision applications," in Proc. IEEE Int. Symp. Neural Networks, vol. 2, June 1996, pp. 874-879.

[10] A. Mhani, G. Sicard, and G. Bouvier, "Analog vision chip for sensing edges contrasts and motion," in Proc. IEEE Int. Symp. Circuits and Systems, vol. 4, Aug. 1997, pp. 2769-2772.

[11] R. E. Cummings, J. V. der Spiegel, P. Mueller, and M. Z. Zhang, "A foveated silicon retina for two-dimensional tracking," IEEE Trans. Circuits Syst. II, vol. 47, pp. 504-517, June 2000.

[12] J. H. Huang, Z. H. Liu, M. C. Jeng, K. Hui, M. Chan, P. K. Ko, and C. Hu, BSIM3 Version 3.2.2 MOSFET Model User's Manual, 1999.

[13] R. A. Boie and W. Turin, "Noise-limited reading of bar codes," IEEE Trans. Ind. Electron., vol. 44, no. 6, pp. 816-824, Dec. 1997.

[14] C. Y. Wu and C. T. Chiang, "A low-photocurrent CMOS retinal focalplane sensor with pseudo-BJT smoothing network and adaptive current Schmitt trigger for scanner applications," in Proc. IEEE 2nd Int. Conf. Sensors, vol. 2, Oct. 2003, pp. 1147-1152.

[15] K. A. Boahen, "A retinomorphic vision system," IEEE Micro, vol. 16, pp. 30-39, Oct. 1996.

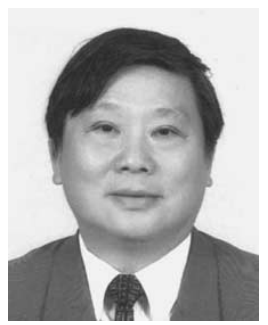

Chung-Yu Wu (S'76-M'76-SM'96-F'98) was born in 1950. He received the M.S. and Ph.D. degrees from the Department of Electronics Engineering, National Chiao-Tung University (NCTU), Hsinchu, Taiwan, R.O.C., in 1976 and 1980, respectively.

Since 1980, he has served as a Consultant in the high-tech industry and research organizations and has built up strong research collaborations with high-tech industries. From 1980 to 1983, he was an Associate Professor at NCTU. From 1984 to 1986, he was a Visiting Associate Professor in the Department of Electrical Engineering, Portland State University, Portland, OR. Since 1987, he has been a Professor at NCTU. From 1991 to 1995, he was rotated to serve as the Director of the Division of Engineering and Applied Science on the National Science Council, Taiwan. From 1996 to 1998, he was honored as the Centennial Honorary Chair Professor, NCTU. In addition, he conducted postdoctoral research at UC Berkeley in summer of 2002. He has published more than 250 technical papers in international journals and conferences. He also holds 19 patents, including nine U.S. patents. His research interests are nanoelectronics and VLSI, including circuits and systems in low-power/low-voltage mixed-signal design, and systems, biochips, neural vision sensors, RF circuits, and CAD analysis.

Dr. Wu is a member of Eta Kappa Nu and Phi Tau Phi Honorary Scholastic Societies. He was a recipient of the IEEE Fellow Award in 1998 and the Third Millennium Medal in 2000. In Taiwan, he received numerous research awards from the Ministry of Education, National Science Council, and professional foundations. 


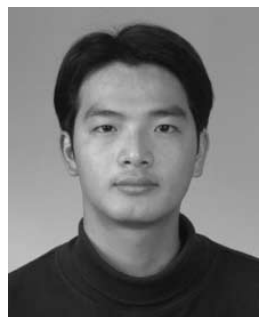

Cheng-Ta Chiang ( $\left.\mathrm{S}^{\prime} 00\right)$ was born in Taiwan, R.O.C., in 1977. He received the B.S. degree in electronics engineering from Chung-Yuan Christian University, Taiwan, and the M.S. degree in biomedical engineering from the National Cheng-Kung University, Taiwan, in 1999 and 2001, respectively. $\mathrm{He}$ is currently pursuing the Ph.D. degree at the Institute of Electronics, National Chiao-Tung University, Hsinchu, Taiwan.

His main research interests include analog neuromorphic vision chips

integrated circuits, biomedical electronics, and 